\title{
Case Report: Potocki-Lupski Syndrome in Five Siblings
}

\author{
Alina Grama ${ }^{1,2 t}$, Claudia Sîrbe ${ }^{1,2 \dagger}$, Diana Miclea ${ }^{3 *}$, Simona Sorana Căinap ${ }^{1,2+}$, \\ Delia Huniadi ${ }^{4}$, Bogdan Bulata ${ }^{5}$ and Tudor Lucian Pop ${ }^{1,2 *}$
}

${ }^{1}$ Second Pediatric Discipline, Department of Mother and Child, University of Medicine and Pharmacy luliu Hațieganu, Cluj-Napoca, Romania, ${ }^{2}$ Second Pediatric Clinic, Emergency Clinical Hospital for Children, Cluj-Napoca, Romania, ${ }^{3}$ Discipline of Medical Genetics, Department of Molecular Sciences, University of Medicine and Pharmacy luliu Hațieganu, Cluj-Napoca, Romania, ${ }^{4}$ Discipline of Neurology, University of Medicine and Pharmacy luliu Hațieganu, Cluj-Napoca, Romania, ${ }^{5}$ Pediatric Nephrology, Dialysis and Toxicology Clinic, Emergency Clinical Hospital for Children, Cluj-Napoca, Romania

\section{OPEN ACCESS}

Edited by:

Rianne Oostenbrink

Erasmus Medical Center, Netherlands

Reviewed by:

Piero Pavone,

University of Catania, Italy

Orkun Tolunay,

Ministry of Health, Turkey

*Correspondence:

Tudor Lucian Pop

tudor.pop@umfcluj.ro

Diana Miclea

diana.miclea@umfcluj.ro

tThese authors have contributed equally to this work and share first authorship

Specialty section:

This article was submitted to

General Pediatrics and Pediatric

Emergency Care,

a section of the journal

Frontiers in Pediatrics

Received: 21 April 2021 Accepted: 07 October 2021 Published: 08 November 2021

Citation:

Grama A, Sirbe $C$, Miclea $D$,

Căinap SS, Huniadi D, Bulata $B$ and

Pop TL (2021) Case Report:

Potocki-Lupski Syndrome in Five

Siblings. Front. Pediatr. 9:698629.

doi: 10.3389/fped.2021.698629
Potocki-Lupski syndrome (PTLS) is a rare developmental disorder resulting from the partial duplication of the short arm of chromosome 17. Affected children may have hypotonia, facial dysmorphism, or neurological abnormalities. PTLS is also frequently associated with failure to thrive due to swallowing difficulties or growth hormone deficiency. We report the first Romanian family (a mother and her five children) diagnosed with PTLS (17p11.2 microduplication). Fortunately, they present a less severe form of the disease. The neurological manifestations (speech delay, mild intellectual disability) are associated with craniofacial dysmorphism (microcephaly, micrognathia, triangular face, broad forehead, long chin, prominent ears, dolichocephaly, down slanting palpebral fissures). The diagnostic was established using a multiplex ligation-dependent probe amplification technique (MLPA) test, which detected the duplication of three regions of the 17p11.2 chromosome (RAl1, DRC3-6, LLGL1-4RA). Children with PTLS have specific phenotypes (craniofacial dysmorphism or neurological manifestations), which must draw the pediatrician's attention to a possible genetic condition. However, every child with this disease is unique and may have a different clinical presentation. A multi-disciplinary team is needed for the management of these patients. The parent's counseling and genetic advice are essential for a family with children with PTLS.

Keywords: children, craniofacial dysmorphism, hypotonia, cognitive delay, 17p112 duplication, Potocki-Lupski syndrome

\section{INTRODUCTION}

Potocki-Lupski syndrome (PTLS) is a very rare chromosomal anomaly (1:25.000 people worldwide), resulting from the partial duplication of the short arm of chromosome 17 (17p11.2 microduplication) (1). PTLS was first reported in 2007, although the first case described appears to be from 1996. The name of the disease is associated with the two researchers who described it, Lorraine Potocki and James R. Lupski (2). PTLS is a developmental disorder with features ranging from mild to severe ones. It is characterized by hypotonia, developmental and intellectual delay, or congenital anomalies. The variability of the clinical presentations is more important regarding the cognitive level and behavioral disorders (3).

Children with PTLS have a characteristic phenotype with facial dysmorphism and neurological or behavioral abnormalities. The craniofacial dysmorphic features include the triangular or oval 
face, micrognathia (in early childhood), high-arched palate, down slanting palpebral fissures, broad forehead, protruding nose, smooth chin, and dental malocclusion (1, 4). The neurological manifestations are variable and give the prognosis of the disease. These children may present speech and language impairment, delayed developmental milestones, intellectual disability, behavioral disorders (repetitive behaviors, anxiety, withdrawal, attention deficit hyperactivity disorder-ADHD, autism spectrum disorders-ASD), motor clumsiness/coordination impairment, or other neuropsychiatric disorders (1, 3, 5). The natural course of PTLS involves hypotonia, feeding difficulties in infants, and sleep disturbance (obstructive or central sleep apnea), more evident as the child gets older, without common anomalies revealed on brain MRI $(1,3,4)$.

In most cases, the genetic abnormality is de novo, and less often, the transmission is autosomal dominant (1). There are few genes involved in PTLS etiopathogenesis, RAI1, SREBF1, DRG2, LLGL1, SHMT1, or ZFP179 (6). The diagnosis of PTLS is established by detecting duplication that encompasses the RAI1 (retinoic acid 1) gene at chromosome 17p11.2. This duplicated region may contain several genes, but RAI1 underlies the main features of PTLS. The recurrent duplication accounts for approximately two-thirds of 17p11.2 duplications and nonrecurrent duplications for about one-third of cases. An additional copy of the RAI1 gene underlies many of the characteristic features of PTLS (7). Also, RAI1 controls the expression of genes involved in the sleep-wake cycle, thus explaining sleep disorders in children with PTLS. But the involvement of the RAI1 gene in triggering intellectual disabilities and neurological manifestations has not been proven. Most likely, they are the result of duplication of the other genes involved.

The exact incidence of these disorders is unknown. More than 50 children with PTLS have been described in the literature so far (1).

\section{CASE PRESENTATION}

We report a family (five children and a mother) with $17 \mathrm{p} 11.2$ microduplication. As far as we know, this is the first described family with PTLS in Romania. The children have three different fathers. The written informed consent was obtained from the children's mother (as sole legal guardian) to publish the cases and any accompanying images. The pedigree analysis of this family is presented in Figure 1.

The diagnostic was established when the fourth brother (child 4) was referred to us at the age of 2 years and 10 months for an acute respiratory infection. He was born at 37 th week of gestation from an unmonitored pregnancy, vaginal delivery. The mother was 33 years old, from a poor background, and with a liminal intellect. None of the pregnancies have been monitored, but the mother denied infections or other problems. At first admission in our unit, the child's physical examination revealed facial dysmorphic features, neurological disorder, and severe malnutrition due to feeding difficulties. The boy's weight was $7.3 \mathrm{~kg}(-3.7$
SD), height $75 \mathrm{~cm}(-3.75 \mathrm{SD})$, and head circumference $42 \mathrm{~cm}$ (-2.7 SD). His facial dysmorphism was impressive: triangular face, micrognathia, dolichocephaly, long chin, down slanting palpebral fissures, and bulbous nose tip. His ears were very prominent, which gave him the appearance of an elf (Figure 2). The neurological examination showed mild axillary hypotonia, speech and language impairment, and developmental delay (Table 1). We performed genetic testing, including the karyotype and multiplex ligation-dependent probe amplification technique (MLPA) for the main microdeleted/microduplicated regions (using SALSA MLPA Probe mix P245 Microdeletion Microduplication Syndromes kit). MLPA test showed the duplication of three regions (RAI1, DRC3-6, LLGL1-4RA) of 17 p11.2 chromosomes corresponding to PTLS.

Subsequently, the other four siblings (an older sister 13 years old, a girl 8 years 2 months old, the youngest girl, 5 years 5 months old, and the smallest brother, aged 3 months) were evaluated. There were no records of the medical history or developmental milestones for the children of this family as they were not registered to a family physician. All children had the same typical disease manifestations (facial dysmorphism, dolichocephaly, prominent ears, and neurologic disorder (Figure 3; Table 1). Also, feeding difficulties and axial hypotonia were observed, especially in the two younger brothers. No sleep disorders or behavioral problems were reported in these children, other than ADHD in the older one. On their first clinical examination, three siblings were underweight, and one child also had short stature (z-score $-2.17 \mathrm{SD}$ ). All brothers were genetically tested by MLPA analysis, and all of them presented 17p11.2 microduplication (RAI1, DRC3-6, LLGL14RA). The duplication was also detected in the peripheral blood of their mother, suggesting the autosomal dominant transmission. The cardiology exam (including electrocardiogram and echocardiography) revealed no pathological changes in all children. Also, abdominal ultrasound examination (including renal examination) and laboratory parameters (electrolytes, liver or renal function tests, and bone metabolism) were in normal ranges.

Even though they have a mild form of PTLS, presenting just some of the clinical features described in this disease, the children are followed now by a multi-disciplinary team including pediatricians, a nutritionist, a neurologist, a psychiatrist, a psychologist, speech therapy specialists, and social-aid workers. We aim to improve their prognostic for the long-term and to minimize intellectual and behavioral problems.

\section{DISCUSSIONS}

We presented a mother and her five children (three girls and two boys) diagnosed with PTLS (17p11.2 microdeletion). All of them had the main clinical manifestations of the disease, respectively craniofacial dysmorphism and neurological impairment. Fortunately, none of them had heart damage, kidney or osteoarticular anomalies, or sleep disorders, often described in patients with the disease (Table 1). Only one child was diagnosed with $\mathrm{ADHD}$, but the others report no behavioral problems. 


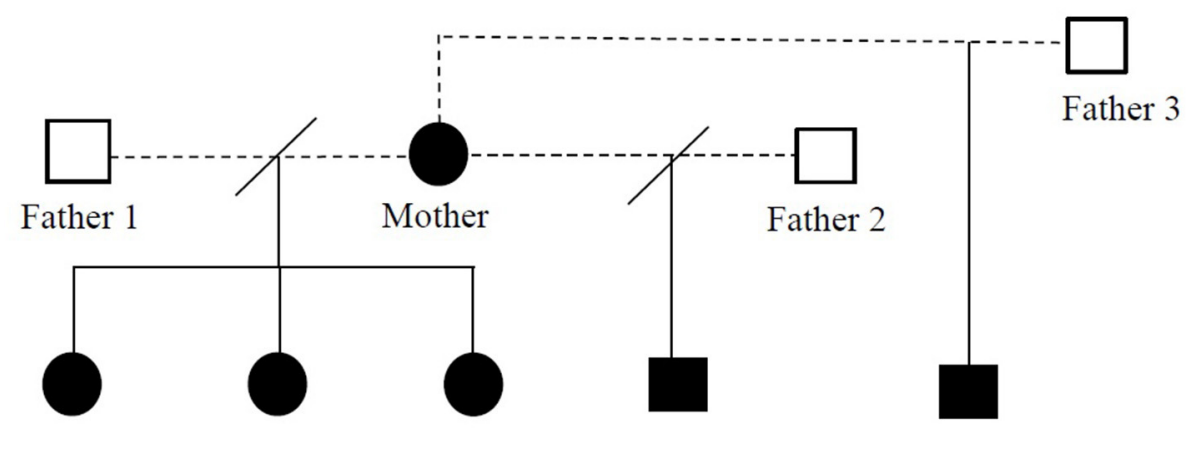

Child $1 \quad$ Child 2 Child $3 \quad$ Child $4 \quad$ Child 5

FIGURE 1 | Pedigree chart of the reported family with Potocki-Lupski syndrome.
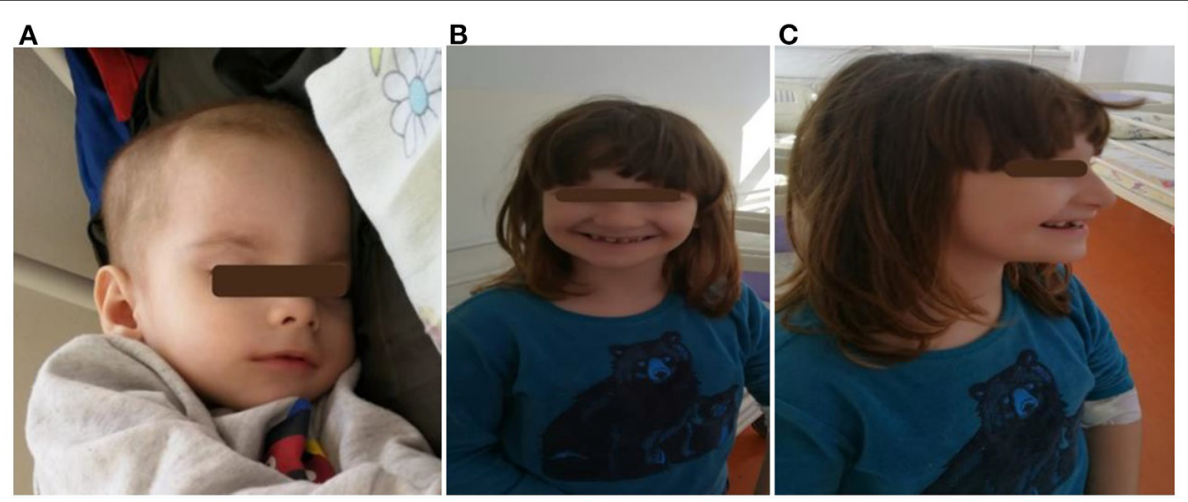

FIGURE 2 | Two siblings: (A) a 3 month-old infant (child 5) with craniofacial dysmorphism; (B,C) a 5- year 5-month-old girl (child 3) with craniofacial dysmorphism, mandibular hypoplasia, dental abnormalities, malocclusion, and intellectual disability.

Duplication 17 p11.2 syndrome is also frequently associated with failure to thrive, as seen in our patients (three of the five children had a growth deficit at their first assessment). Malnutrition may be due to gastroesophageal reflux or swallowing difficulties (dysphagia), leading to feeding problems during infancy. In one of our children, the height was also reduced. Short stature may be due to severe malnutrition or growth hormone deficiency when it is often associated with hypoglycemia $(1,2,4,8,9)$.

Congenital malformations may be present in children with PTLS. Cardiovascular anomalies (dilated aortic root, bicommissural aortic valve, atrial/ventricular, and septal defects) are described in $\sim 40 \%$ of children with PTLS. The connective tissue damage found in children with PTLS explains a more frequent aorta or aortic valve involvement, the most common abnormality being aortic root dilation (8). Congenital heart defects can be severe and progress to severe heart failure, requiring heart transplantation. In 2011, the first case with PTLS with heart transplantation had been reported: a 10 -yearold boy with 17p11.2 duplication and hypoplastic left heart syndrome (9). Genetic factors determine the cardiovascular damage in PTLS. Our children had no signs of structural heart disease, aortic or valvular disorders, but they need periodic cardiovascular monitoring given the risk for cardiovascular involvement. Kidney anomalies have been observed in over $10 \%$ of affected individuals. The most common are hypoplastic kidneys, hydronephrosis, or multi-cystic dysplastic kidneys. Ocular, musculoskeletal, and osteoarticular abnormalities are also described but less frequently $(4,6-9,11)$.

PTLS may be suspect based on the child's appearance. The phenotype is often not so suggestive for this disease. Genetic testing must be used in a patient with an intellectual disability or other psychiatric or neurologic signs associated with facial dysmorphism, congenital anomalies and/or failure to thrive. In 2013, the first case of PTLS with the prenatal diagnosis was described. It was a fetus with hypoplastic left heart and aberrant right subclavian artery in which fetal karyotype was performed by amniocentesis (10).

Differential diagnoses of PTLS include Smith-Magenis syndrome (SMS), Down syndrome, Williams syndrome, brachydactyly-intellectual deficit syndrome (del 2q37), PraderWilli syndrome, or Sotos syndrome. The first condition that needs to be excluded is SMS, a rare syndrome with similar clinical manifestations and chromosome 17 involvement (deletion or 
TABLE 1 | Clinical and genetic data of the reported family compared to reported features in children with Potocki-Lupski syndrome (1-10).

\begin{tabular}{|c|c|c|c|c|c|c|}
\hline Clinical and genetic data & Child 1 & Child 2 & Child 3 & Child 4 & Child 5 & Mother \\
\hline Age at diagnosis & 13 yrs & $\begin{array}{c}8 \text { yrs, } 2 \\
\text { mos }\end{array}$ & $\begin{array}{c}5 \text { yrs, } 5 \\
\text { mos }\end{array}$ & $\begin{array}{c}2 \mathrm{yrs}, 10 \\
\text { mos }\end{array}$ & 3 mos & 33 yrs \\
\hline Genetic test (MLPA) & \multicolumn{6}{|c|}{ 17p11.2 microduplication (RAl1, DRC3-6, LLGL1-4) } \\
\hline $\begin{array}{l}\text { Malnutrition } \\
\text { BMl z-score }\end{array}$ & $\begin{array}{c}\text { No } \\
0.2 \mathrm{SD}\end{array}$ & $\begin{array}{c}\text { Yes } \\
-2.4 \text { SD }\end{array}$ & $\begin{array}{c}\text { Yes } \\
-2.8 \mathrm{SD}\end{array}$ & $\begin{array}{c}\text { Yes } \\
-3.7 \mathrm{SD}\end{array}$ & $\begin{array}{c}\text { No } \\
0.12 \mathrm{SD}\end{array}$ & $\begin{array}{c}\text { No } \\
0.5 \mathrm{SD}\end{array}$ \\
\hline
\end{tabular}

Facial features

Micrognathia

Down-slanting palpebral fissures

Broad forehead

Long nasal tip

Triangular face

The prominence of the angle of the jaw

Oval-shape face and longer chin

Microcephalia

Hypertelorism

Low-set and posteriorly rotated ears

Asymmetric smile

Neurodevelopmental features

Mild-to-moderate infantile hypotonia

Oropharyngeal dysphagia

Poor feeding

Mild-to-moderate gross motor delays

Cognitive impairment

Developmental milestones delay

peech delay (expressive and receptive language impairment,

articulation difficulties, disordered intonation, prosody)

Verbal apraxia

Sleep-disordered breathing (mild central and/or obstructive

sleep apnea)

Behavioral difficulties (attention deficit, withdrawal,

hyperactivity, anxiety, ADHD)

Autism spectrum disorders (ASD)

Autistic features (decreased eye contact, motor mannerisms, posturing, sensory hypersensitivity, repetitive behaviors,

difficulties with transitions, lack of appropriate functional or symbolic play, lack of joint attention)

Congenital heart disease

Atrial septal defect

Ventricular septal defect

Bicuspid aortic valve

Dilated aortic root

Hypoplastic left heart

Arrhythmia

Growth hormone deficiency

Short stature

Hypoglycemia

Musculoskeletal features

Severe bilateral clubfoot

Joint hypermobility

Kyphoscoliosis

Flat foot

Long fingers and toes

Renal anomalies

Hypoplastic kidneys

Multi-cystic dysplastic kidneys

Hydronephrosis

\section{e}


TABLE 1 | Continued

\begin{tabular}{|c|c|c|c|c|c|c|}
\hline Clinical and genetic data & Child 1 & Child 2 & Child 3 & Child 4 & Child 5 & Mother \\
\hline \multicolumn{7}{|l|}{ Others } \\
\hline Hyperopia & - & - & - & - & - & - \\
\hline Mild high-frequency sensorial hearing loss & - & - & - & - & - & - \\
\hline Dental malocclusion or dental crowding & + & + & + & - & - & + \\
\hline
\end{tabular}

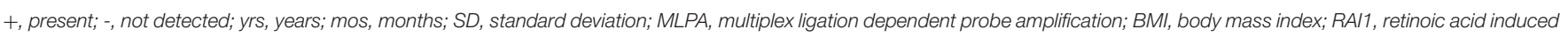
1; DRC, dynein regulatory complex; LLGL, lethal giant larvae homolog.

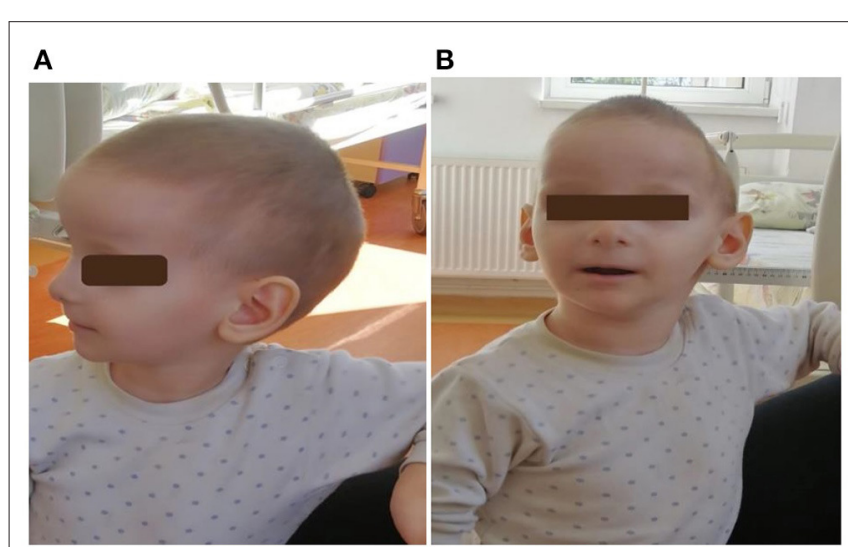

FIGURE 3 | (A,B): First patient (child 4) at 2 years and 10 months. He had craniofacial dysmorphism, ears with thin helices, deep conchae, and hypoplastic lobules.

a mutation in the RAI1 gene) (12). Both PTLS and SMS occur because of a non-allelic homologous recombination defect involving a $1.3-3.7 \mathrm{Mb}$ of $17 \mathrm{p} 11.2$ chromosomal region in which the RAI1 gene is found. Other genes that have been identified in this region are SREBF1, DRG2, LLGL1, SHMT1, and ZFP179 (12-14).

There is no specific treatment for children with PTLS. Each child will benefit from an individualized treatment adapted to his needs, including speech therapy, physical therapy, behavior and communication therapies, or different specialized education services. Managing children with severe forms (congenital heart defects, renal or gastrointestinal disorders) requires a multidisciplinary team. Collaboration between specialists in pediatrics, cardiology, genetic disease, neurology, psychiatry, psychology, nutrition, nephrology, and orthopedics is mandatory in caring for these children $(13,14)$. Genetic advice is essential in such cases. After the first four children's diagnosis, the mother (who was already pregnant with the 5 th child) was informed about the risk of the disease in subsequent pregnancies. With this last pregnancy, she decided to ligate the fallopian tubes.

A limitation in the genetic analysis was the impossibility of the precise delimitation of the region involved in duplication to indicate if it is a recurrent or a non-recurrent one and, therefore, suggesting some correlations between an accurate copy number variant and the phenotype. Also, it was not possible to perform genetic testing by chromosomal microarray for these patients. However, we considered it necessary to report this family with an affected mother, diagnosed only after the birth of all her five affected children. Also, the lack of specificity of the clinical presentation in PTLS indicates the need for a more rigorous evaluation of individuals with intellectual disability (isolated or syndromic) by genetic testing, as chromosomal microarray or MLPA technique (13). An accurate diagnosis of the mother before pregnancies would have been beneficial for providing timely genetic counseling, including the high risk of recurrence (50\%), thus helping the mother and her family make an informed decision regarding future pregnancies.

\section{CONCLUSIONS}

Children with PTLS are at a high risk of neurodevelopmental disorders (especially learning and language disabilities) or ASD, leading to behavior problems or social interaction and communication difficulties. In a child with suggestive phenotypes for a genetic disease, the main aim of the pediatrician is an early diagnosis to prevent possible complications. A multi-disciplinary team is needed for these cases. The parents of these children should be counseled and helped to deal with medical problems, psychological behavior, and neuropsychiatric disorders that may occur because the prognosis of these children is difficult to predict. Moreover, extremely important is genetic advice.

\section{DATA AVAILABILITY STATEMENT}

The original contributions presented in the study are included in the article, further inquiries can be directed to the corresponding authors.

\section{ETHICS STATEMENT}

Written informed consent has been obtained from the parents of the patients to publish this paper. Written informed consent to participate in this study was provided by the participants' legal guardian/next of kin. Written informed consent was obtained from the minors' sole legal guardian (mother) for the publication of any potentially identifiable images or data included in this article.

\section{AUTHOR CONTRIBUTIONS}

AG, DM, and TLP: methodology. AG, CS, DM, SSC, $\mathrm{BB}, \mathrm{DH}$, and TLP: formal analysis. AG, CS, and TLP: 
writing-original draft preparation. AG, CS, DM, and TLP: writing-review and editing. All authors

\section{REFERENCES}

1. Sumathipala DS, Mandawala EN, Sumanasena SP. et al. $17 \mathrm{p} 112$ and Xq28 duplication detected in a girl diagnosed with Potocki-Lupski syndrome. BMC Res Notes. (2015) 8:506. doi: 10.1186/s13104-015-1439-7

2. Potocki L, Bi W, Treadwell-Deering D, Carvalho CM, Eifert A, Friedman EM et al. Characterization of Potocki-Lupski syndrome (dup (17) (p11.2p112)) and delineation of a dosage-sensitive critical interval that can convey an autism phenotype. Am J Hum Genet. (2007) 80:633-49. doi: 10.1086/512864

3. Ciaccio C, Pantaleoni C, Milani D, Alfei E, Sciacca FL, Canafoglia L, et al. Neurological phenotype of Potocki-Lupski syndrome. Am J Med Genet A. (2020) 182:2317-24. doi: 10.1002/ajmg.a.61789

4. Praticò AD, Falsaperla R, Rizzo R, Ruggieri M, Verrotti A, Pavone P, et al. New patient with Potocki-Lupski syndrome: a literature review. J Pediatr Genet. (2018) 7:29-34. doi: 10.1055/s-0037-1604479

5. Bissell S, Wilde L, Richards C, Moss J, Oliver C. The behavioural phenotype of Potocki-Lupski syndrome: a cross-syndrome comparison. J Neurodev Disord. (2018) 10:2. doi: 10.1186/s11689-017-9221-x

6. Shuib S, Saaid NN, Zakaria Z, Ismail J, Abdul Latiff Z. Duplication 17p11. 2 (Potocki-Lupski Syndrome) in a child with developmental delay Malays. $J$ Pathol. (2017) 39:77-81.

7. Potocki L, Neira-Fresneda J, Yuan B. "Potocki lupski syndrome," In: Adam MP, Edinger HH, Pagon RA, et al. editors GeneReviews $($ [Internet]. Seattle, WA: University of Washington. (2017). P. 1993-2021.

8. Jefferies JL, Pignatelli RH, Martinez HR, Robbins-Furman PJ, Liu P, Gu W, et al. Cardiovascular findings in duplication 17p11. 2 syndrome. Genet Med. (2012) 14:90-4. doi: 10.1038/gim.0b013e3182329723

9. Sanchez-Valle A, Pierpont ME, Potocki L. The severe end of the spectrum: hypoplastic left heart in Potocki-Lupski syndrome. Am J Med Genet A. (2011) 155:363-6. doi: 10.1002/ajmg.a.3 3844

10. Bravo C, Gámez F, Pérez R, Águarón A, León-Luis JD. Prenatal diagnosis of Potocki-Lupski syndrome in a fetus with hypoplastic left heart and aberrant right subclavian artery. J Perinatol. (2013) 33:394-6. doi: 10.1038/jp.2012.77 have read and agreed to the published version of the manuscript.
11. Soler-Alfonso C, Motil KJ, Turk CL, Robbins-Furman P, Friedman EM, Zhang F, et al. Potocki-Lupski syndrome: a microduplication syndrome associated with oropharyngeal dysphagia and failure to thrive. J Pediatr. (2011) 158:655659.e2. doi: 10.1016/j.jpeds.2010.09.062

12. Girirajan S, Vlangos C, Szomju B, Edelman E, Trevors CD, Dupuis L. et al. Genotype-phenotype correlation in Smith-Magenis syndrome: evidence that multiple genes in $17 \mathrm{p} 112$ contribute to the clinical spectrum. Genet Med. (2006) 8:417-27. doi: 10.1097/01.gim.0000228215.32110.89

13. Potocki L, James R, Stankiewicz P. Genomic disorders: molecular mechanisms for rearrangements and conveyed phenotypes. PLoS Genet. (2007) 1:e49. doi: 10.1371/journal.pgen.0010049

14. Molina J, Carmona-Mora P, Chrast J, Krall PM, Canales CP, Lupski JR, et al. Abnormal social behaviors and altered gene expression rates in a mouse model for Potocki-Lupski syndrome (PDF). Hum Mol Genet. (2008) 17:248695. doi: $10.1093 / \mathrm{hmg} / \mathrm{ddn} 148$

Conflict of Interest: The authors declare that the research was conducted in the absence of any commercial or financial relationships that could be construed as a potential conflict of interest.

Publisher's Note: All claims expressed in this article are solely those of the authors and do not necessarily represent those of their affiliated organizations, or those of the publisher, the editors and the reviewers. Any product that may be evaluated in this article, or claim that may be made by its manufacturer, is not guaranteed or endorsed by the publisher.

Copyright (๑) 2021 Grama, Sìrbe, Miclea, Căinap, Huniadi, Bulata and Pop. This is an open-access article distributed under the terms of the Creative Commons Attribution License (CC BY). The use, distribution or reproduction in other forums is permitted, provided the original author(s) and the copyright owner(s) are credited and that the original publication in this journal is cited, in accordance with accepted academic practice. No use, distribution or reproduction is permitted which does not comply with these terms. 